\title{
Erratum to: Streptomyces mangrovi sp. nov., isolated from mangrove forest sediment
}

\author{
Ghada Yousif • Kanungnid Busarakam • \\ Byung-Yong Kim · Michael Goodfellow
}

Published online: 1 October 2015

(C) Springer International Publishing Switzerland 2015

\section{Erratum to: Antonie van Leeuwenhoek (2015) \\ 108:783-791, DOI 10.1007/s10482-015-0533-0}

Later to the publication of the article, another Streptomyces species has recently been named as Streptomyces mangrovi. We here propose a replacement name for the taxon represented by strain $\mathrm{GY}^{\mathrm{T}}$ and its description is given below.

\section{Description of Streptomyces mangrovicola sp. nov.}

Streptomyces mangrovicola (man.gro.vi'co.la. N.L.mangrovum mangrove; L. suff -cola (from L.n. incola) an inhabitant; N.L. n. mangrovicola, mangrove inhabitant).

Aerobic, Gram-positive, non-acid-alcohol fast actinomycete which forms an extensively branched

The online version of the original article can be found under doi:10.1007/s10482-015-0533-0.

G. Yousif · K. Busarakam · M. Goodfellow $(\square)$

School of Biology, University of Newcastle,

Newcastle upon Tyne NE1 7RU, UK

e-mail: m.goodfellow@ncl.ac.uk

G. Yousif

Botany Department, Faculty of Science, Beni-Suef

University, Beni-Suef, Egypt

\section{B.-Y. Kim}

ChunLab Inc., Seoul National University, Seoul 151-742,

Korea substrate mycelium which carries aerial hyphae that differentiate into spiral chains of rough to warty ornamented spores on yeast extract-malt extract agar. Melanin pigments are not formed on peptone-yeast extract or tyrosine agars. Grows from 10 to $45{ }^{\circ} \mathrm{C}$, optimally $\sim 28^{\circ} \mathrm{C}$, from $\mathrm{pH} 7.0$ to 11 , optimally $\sim \mathrm{pH}$ 7.0 , and in the presence of $10 \%$, w/v synthetic sea salt. Obligate requirement for seawater. Tweens 20 and 60, and xylan are degraded, but not adenine, hypoxanthine, L-tyrosine or xanthine. Hydrolyses aesculin and arbutin and produces $\mathrm{H}_{2} \mathrm{~S}$. Grows on L-arabinose, dextrin, glycogen, L-lactose, D-maltose, D-mannose, D-melibiose, L-ribose, D-salicin, D-sorbose and D-sucrose as sole carbon sources, but not on xylitol (all at $1 \%, \mathrm{w} / \mathrm{v}$ ). $\mathrm{L}$-arginine, L-asparagine and L-valine are used as sole carbon and nitrogen sources, but not L-alanine, Laminobutyric acid, L-cysteine, L-hydroxyproline, Lisoleucine, DL-methionine, L-norvaline, L-ornithine, Lphenylalanine, L-proline, L-serine or L-tryptophan as sole nitrogen sources (all at $0.1 \%$, w/v). Additional phenotypic features are cited in the main text and in Tables 1 and 2 of the original paper. Chemotaxonomic properties are typical of the genus Streptomyces.

The type strain, GY1 ${ }^{\mathrm{T}}\left(=\mathrm{NCIMB} 14980^{\mathrm{T}}=\mathrm{NRRL}\right.$ B-69296 ${ }^{\mathrm{T}}$ ) was isolated from the sediment of the mangrove plant Avicennia mariana at Wadi El Jimal on the Red Sea coast in Egypt. The species description is based on a single strain and thereby serves as a description of the type strain. The GenBank accession number for the 16S rRNA sequence of strain $\mathrm{GY}^{\mathrm{T}}{ }^{\mathrm{T}}$ is $\mathrm{KP} 221800$. 